\title{
Intra Peritoneal Chemotherapy in Epithelial Ovarian Cancers
}

\author{
SOMASHEKHAR S. P.
}

Epithelial Ovarian cancers is called the 'Silent Killer". Unfortunatley most of the women world wide and even in India come to us in Stage III The current standard treatment is Optimal cytoreduciton followed by 6 cycles of IV Paclitaxel and Carboplatin chemotherapy. The sad reality is median progression free survival (PFS) is 16 months, \& median overall survival (OS) is 40 months. Proportion of long term survivors is static. There is no improvement by adding additional drugs. Peritoneal cavity is the principle site of disease in ovarian cancer. The rational for IP chemotherapy is that the peritoneal cavity, the predominant site of tumour, receives sustained exposure to high concentrations of antitumour agents, while normal tissue to bone marrow, are relatively spared. IP administration of chemotherapy was first proposed nearly three decades ago by Dedrick . Compared to intravenous (IV) treatment, intraperitoneal (IP) administration of chemotherapeutic agents permits a severalfold increase in drug concentration to be achieved within the abdominal cavity. The pharmocological advantage of the intraperitoneal route for drugs such as cisplatin and paclitaxel is considerable,with intraperitoneal-to-plasma concentration ratios in the range of more than 20 and 1000 , respectively.

This route allows the escalation of the dose of chemotherapy to a level that is not possible to achieve safely with intravenous drug

Surgical \& Gynec. Oncologist Manipal Comprehehsive Cancer Center Manipal Hospital, Airport Road, Bangalore-560 017 India

E-mail: somusp@yahoo.com administration. Drugs are delivered by the intraperitoneal route only to a depth of few millimeters beneath the tumour surface.

Thus patients with small volume residual disease(i.e.optimally debulked)are expected to benefit most from this approach.

Over 7 randomized trials during last 10 years of IV vs. IP chemotherapy, have shown hazard ratio (HR) for both progression and death reduced 0.80 (Cochrane analysis) which translates into a 12 month increase in median survival. GOG 111, study changed the standard of care "Overnight" and showed , 5 month difference in PFS of 13 vs.18 months, with 12 month difference in OS of 24 vs. 36 months 3 prospective randomized trials, recent one's is from Armstrong et. al, \& Markman, et.al, and Albert's et. al, have shown survival benefit from IP chemotherapy.

In most of the trial the IP chemoport catheter related complications is in the tune of $40 \%$. Hence the importance of placing the right type of IP chemoport catheter and the right technique of placement.

There are lot of proponents as well as criticizers for IP chemoport. The advantages outlined are, that it gives higher PFS and overall survival (OS), is easy to adminster. But the points against are that, complications are high with IP administration and catheter related complications are also high and only over $45 \%$ of patients are able to complete prescribed cycles of IP chemotherapy and more visit to hospital is required for IP regimen Till now ideal regimen of IP chemotherapy has not been 
defined. Certain modifications to IP regimen in the form of Pactitaxel on day 1 given over 3 hours instead of 24 hours and day ${ }^{2}$ to Cisplatin dose reduced from $100 \mathrm{mg} / \mathrm{m} 2$ to $75 \mathrm{mg} / \mathrm{m} 2$ and day 8 Paclitaxel of $60 \mathrm{mg} / \mathrm{m} 2$, and good chemoport catheter placement techniques and using appropriate catheters, reduce these toxities and most of the patients are able to complete prescribed dose and cycles. The option of replacing Cisplatin by carboplatin is very attractive as far as toxicity profiles is concerned.

In Manipal Comprehensive Caner Center, Bengaluru, since Jan 2007 till to date, we have used Bard IP chemoport intraoperatively for Optimally cytoreduced Stage IIIc EOC. Total of 22 patients received IP chemotherapy and 8 patients of them received after Optimal cytoreduction. Except one patient all the patients are able to complete prescribed cycles of IP chemotherapy. Only one patiens who had Cisplatin induced toxicities, received IP carboplatin successfully. There were no major catheter related complications. And till to date all patients are on follow up are doing well.

\section{Conclusion:}

IP chemotherapy is feasible, with accepted toxity and gives long term PFS and OS. Even in Indian patients intraperitonael chemotherapy is feasible with acceptable toxicity. IP chemoport cathteter related complications are minimal. Most of the patients are able to complete all cycles of IP chemotherapy. More Randomized control trials will be required to practice this protocol. Serious discussion with optimally cytoreduced stage IIIc EOC patients is required to offer IP chemotherapy as option as part of their treatment. 